\title{
SYMMETRY AND UNIFORM APPROXIMATION BY ANALYTIC FUNCTIONS
}

\author{
DMITRY KHAVINSON
}

(Communicated by Irwin Kra)

\begin{abstract}
In this paper we treat the problem of finding all the domains in C for which the uniform distance from the function $\bar{z}$ to the space of analytic functions is equal precisely to ( 2 area/perimeter). We show that for simply connected domains it occurs if and only if the domain is a disk. We also discuss the relation of the above problem to certain types of symmetry in potential theory and to the theory of Schwarz functions.
\end{abstract}

1. Introduction. Let $G$ be a finitely connected region in $\mathbf{C}$ bounded by $n$ simple closed analytic curves $\gamma_{j}, j=1, \ldots, n$, i.e. $\Gamma=\partial G=\bigcup_{j=1}^{n} \gamma_{j}$. Let $A(G)$ denote the Banach algebra of functions analytic in $G$ and continuous up to the boundary with the usual norm

$$
\|f\|_{\infty}=\|f\|_{A(G)}=\sup _{z \in G}|f(z)| .
$$

It is well known that for such $G, A(G)$ coincides with the uniform closure of the algebra of rational functions with poles outside the closure of $G$. The following quantity, which we shall call analytic content, characterizing $G$ by means of $A(G)$, has been introduced in [9] (also see [6]):

$$
\lambda=\lambda(G) \stackrel{\text { def }}{=} \inf _{\phi \in A(G)}\|\bar{z}-\phi(z)\|_{\infty} .
$$

Making use of Stokes' formula and the Hahn-Banach theorem, it is not hard to show that $\lambda(G)$ can also be expressed in terms of Cauchy potentials of measures orthogonal to $A(G)$. Namely (see [9]),

$$
\lambda(G)=\frac{1}{\pi} \sup _{\substack{\mu \perp A(G) \\\|\mu\| \leq 1}}\left|\iint_{G} \hat{\mu}(z) d A\right|
$$

where $d A=d x d y$ is the area measure in $\mathbf{C}$, and

$$
\hat{\mu}(z)=\int_{\mathbf{C}} \frac{d \mu(\varsigma)}{\varsigma-z}
$$

denotes the Cauchy potential of a complex-valued, finite Borel measure $\mu$ in $\mathbf{C}$.

Received by the editors February 21, 1986 and, in revised form, July 25, 1986.

1980 Mathematics Subject Classification (1985 Revision). Primary 30E10; Secondary 31B35.

The author was partially supported by the National Science Foundation under grant DMS8400582 . 
It turns out that $\lambda(G)$ can be easily estimated in terms of very simple geometric characteristics of $G$ such as area and perimeter. In [2], H. Alexander has shown that for all $G$

$$
(\operatorname{area}(G) / \pi)^{1 / 2} \geq \lambda(G) .
$$

On the other hand, in [9] we have proved that for all $G$

$$
\lambda(G) \geq 2 \operatorname{area}(G) / \text { perimeter }(G) .
$$

One of the surprising corollaries from (1) and (2) is the classical isoperimetric inequality

$$
P^{2} \geq 4 \pi A
$$

where $P=$ perimeter of $G$, and $A=$ area of $G$. For further discussion concerning various forms of (1) and (2) and their affiliation with (3) we refer the reader to [3, 10, 12, and 6]. Here, we only remark that both (1) and (2) are sharp, since they become equalities for disks. In another direction, it is not hard to show [6] that if equality holds in (1), then $G$ must be a disk of radius $\lambda$. However, the extremal case in (2) turns out to be much harder to investigate. In particular, as we show in $\S 3$, equality in (2) also holds when $G$ is an annulus. To our knowledge, the answer to the question whether a disk and an annulus are the only extremal domains for (2) remains unknown.

Let us briefly describe the contents of the rest of the paper.

In $\S 2$ we prove (Theorem 1) that equality held in (2) is equivalent to some symmetry properties of $G$ with respect to potential theory, and also equivalent to certain differential-geometric properties of $\Gamma$ expressed in terms of the "tangential" ordinary differential equation of Riccati type.

In $\S 3$ we show that equality in (2) also holds for annuli.

In $\S 4$, using a deep result of J. Serrin [17] in potential theory, we show that for simply connected regions $G$ equality in (2) only holds for disks.

Finally, $\S 5$ contains a few remarks concerning the results and methods of this paper.

ACKNOWLedgments. The author is grateful to Professor M. Sakai, Professor Harold S. Shapiro, and Professor L. Zalcman for pointing out to him the references $[15,17$, and 18], and to Dr. B. Gustafsson for many enlightening discussions. Also, the author wants to thank the referee for his suggestions, which helped to improve the exposition.

2. A characterization of extremal domains in terms of potential theory and differential geometry.

THEOREM 1. Let $G, \lambda=\lambda(G), A, P$ be as above. The following are equivalent.

(i) $\lambda=2 A / P$.

(ii) $\exists \phi^{*} \in A(G)$ such that the equality

$$
\bar{\zeta}(s)-i \lambda \frac{d \bar{\zeta}}{d s}=\phi^{*}(\varsigma(s))
$$

holds on $\Gamma$. Here, $s$ is the arclength parameter on $\Gamma$. Moreover, $\phi^{*}$ is the best approximation of $\bar{z}$ in $A(G)$, i.e.

$$
\left\|\bar{z}-\phi^{*}(z)\right\|_{\infty}=\lambda
$$


(iii) For all $f \in A(G)$ the following mean value property holds:

$$
\frac{1}{A} \iint_{G} f d A=\frac{1}{P} \int_{\Gamma} f d s .
$$

(iv) (4) holds for all $u \in \operatorname{Re} A(G)$, where $\operatorname{Re} A(G)=\{u: \exists f \in A(G): u=\operatorname{Re} f\}$.

ProOF. Since both measures $d A$ and $d s$ are real-valued, then taking real parts in (4), we find that (iii) $\Leftrightarrow$ (iv). To show that (i) $\Leftrightarrow$ (ii), consider the extremal problem

$$
\lambda \stackrel{\text { def }}{=} \inf _{\phi \in A(G)}\|\bar{z}-\phi(z)\|_{\infty}
$$

As $\bar{z}-\phi$ is harmonic in $G$ for all $\phi \in A(G)$, we have

$$
\lambda=\inf _{\phi \in A(G)}\|\bar{z}-\phi(z)\|_{C(\Gamma)}
$$

where $C(\Gamma)$ is the space of all continuous functions on $\Gamma$ with the usual sup-norm. It is well known (see e.g. [13], also see [5, Chapter VIII]) that there exists a function $\phi^{*} \in H^{\infty}(G)$, for which the infimum is attained. Since $\Gamma$ is analytic, there is a function $S(z)$ analytic in a tubular neighborhood of $\Gamma$ such that $\bar{z}=S(z)$ on $\Gamma .(S(z)$ is called the Schwarz function of $\Gamma$; see $[\mathbf{1}, 4]$.$) Thus, according to$ the $\mathrm{S}$. Ya. Khavinson result on the behavior of extremal functions (see $[13$, Theorem 5.13$]), \phi^{*}$ can be analytically continued across $\Gamma$. In particular, $\phi^{*} \in A(G)$. From the Stokes formula, we find

$$
\int_{\Gamma} \bar{\zeta} d \zeta=2 i \iint_{G} \frac{\partial \bar{\zeta}}{\partial \bar{\zeta}} d A=2 i A
$$

where, as usual, $\partial / \partial \bar{\zeta}=(\partial / \partial \xi+i \partial / \partial \eta) / 2, \varsigma=\xi+i \eta$. Since we assumed that (i) holds, then from (5), using Stokes' formula again and recalling that $\partial \phi^{*} / \partial \bar{\zeta} \equiv 0$ in $G$ as $\phi^{*} \in A(G)$, we obtain the following inequality:

$$
\begin{aligned}
\lambda & =\frac{2 A}{P}=\frac{1}{P}\left|2 i \iint_{G} \frac{\partial}{\partial \bar{\zeta}}\left(\bar{\zeta}-\phi^{*}(\varsigma)\right) d A\right| \\
& =\frac{1}{P}\left|\int_{\Gamma}\left(\bar{\zeta}-\phi^{*}(\varsigma)\right) d \varsigma\right| \leq \frac{1}{P}\left\|\bar{\zeta}-\phi^{*}\right\|_{\infty} \int_{\Gamma} d s=\left\|\bar{\zeta}-\phi^{*}\right\|_{\infty} .
\end{aligned}
$$

Since $\phi^{*}$ is the extremal function, equality must hold in (6). Hence

$$
\left|\int_{\Gamma}\left(\bar{\zeta}-\phi^{*}(\zeta)\right) d \varsigma\right|=\int_{\Gamma}\left|\bar{\zeta}-\phi^{*}(\zeta)\right| d s=P\left\|\bar{\zeta}-\phi^{*}\right\|_{\infty}=P \lambda .
$$

Therefore, the differential $\left(\bar{\zeta}-\phi^{*}(\zeta)\right) d \zeta$ has a constant argument on $\Gamma$ and $\left|\bar{\zeta}-\phi^{*}(\varsigma)\right| \equiv \lambda$ on $\Gamma$. Thus,

$$
e^{i \alpha}\left(\bar{\zeta}-\phi^{*}(\zeta)\right) d \varsigma=\lambda d s
$$

for all $\varsigma \in \Gamma$, where $\alpha$ is a real constant. Using Stokes' formula again, and recalling that $\lambda=2 A / P$, we obtain from (7)

$$
\int_{\Gamma} \lambda d s=2 A=e^{i \alpha} \int_{\Gamma}\left(\bar{\zeta}-\phi^{*}(\zeta)\right) d \zeta=e^{i \alpha} \cdot 2 i A .
$$


Hence, $e^{i \alpha}=-i$. As $d s / d \zeta=d \bar{\zeta} / d s$, we finally conclude from (7) that

$$
\bar{\zeta}-\phi^{*}(\zeta)=i \lambda d \bar{\zeta} / d s
$$

and this is precisely what we had to prove.

To show that (ii) $\Rightarrow$ (iii), let us take $f \in A(G)$. Then from (ii), Cauchy's theorem, and Stokes' formula we obtain

$$
\frac{1}{P} \int_{\Gamma} f d s=\frac{1}{P} \int_{\Gamma} f \frac{d \bar{\zeta}}{d s} d \varsigma=\frac{1}{P} \int_{\Gamma} \frac{1}{i \lambda}\left(\bar{\zeta}-\phi^{*}(\varsigma)\right) f(\varsigma) d \varsigma=\frac{1}{\lambda P} 2 \iint_{G} f d A .
$$

In particular, taking $f \equiv 1$, we find from (8) that $\lambda=2 A / P$. Plugging this back into (8) we obtain (iii).

It remains to show that (iii) $\Rightarrow$ (ii) $\Rightarrow$ (i). Assume that (iii) holds. Take an arbitrary $f \in A(G)$. Using Stokes' formula and (iii) we obtain

$$
\int_{\Gamma} \bar{\zeta} f(\varsigma) d \varsigma=2 i \iint_{G} f d A=\frac{2 i A}{P} \int_{\Gamma} f d s=\frac{2 i A}{P} \int_{\Gamma} f \frac{d \bar{\zeta}}{d s} d \zeta
$$

Since (9) holds for all $f \in A(G)$, the measure $\{\bar{\zeta}-(2 i A / P)(d \bar{\zeta} / d s)\} d \varsigma$ is orthogonal to $A(G)$. Therefore, according to the F. and M. Riesz theorem (see $[14 ; 5$, Chapter X]; also see [11]), there exists a function $\phi^{*}(\varsigma) \in L^{1}(\Gamma, d s)$-closure of $A(G)$ such that

$$
\left[\bar{\zeta}-\frac{2 i A}{P} \frac{d \bar{\zeta}}{d s}\right] d \zeta=\phi^{*}(\varsigma) d \zeta .
$$

But $\bar{\zeta}, d \bar{\zeta} / d s$ are real analytic on $\Gamma$. Hence, $\phi^{*}(\varsigma)$ is also real analytic on $\Gamma$, and therefore its Poisson extension into $G$ is analytic in $G$ and continuous up to $\Gamma$. So, $\phi^{*} \in A(G)$.

Also, from (10) we obtain $\left|\bar{\zeta}-\phi^{*}(\zeta)\right| \equiv 2 A / P$ on $\Gamma$. Hence, $\left\|\bar{\zeta}-\phi^{*}(\zeta)\right\|_{\infty}=2 A / P$. Thus, $\lambda(G) \leq 2 A / P$. As for all $G, \lambda \geq 2 A / P$ (see (2)), we conclude that $\lambda=2 A / P$. This completes the proof of our theorem.

REMARK. Condition (iv) implies that the charge uniformly distributed over $G$ and charge uniformly distributed over $\Gamma$ induce the same electrostatic field at $\infty$. In other words, (iv) implies

$\left(\right.$ iv $\left.^{\prime}\right)$

$$
\iint_{G} \log \frac{1}{|z-\varsigma|} \frac{d A}{A}=\int_{\Gamma} \log \frac{1}{|z-\varsigma|} \frac{d s}{P}
$$

for all $z$ in the unbounded connected component of $\mathbf{C} \backslash \bar{G}$. In reality, for all such $z, \log (1 /|z-\varsigma|)$ is harmonic in $G$ and has a single-valued conjugate there, i.e., $\log (1 /|z-\varsigma|) \in \operatorname{Re} A(G)$. So (iv) $\Rightarrow\left(\right.$ iv $\left.^{\prime}\right)$. On the other hand, it is clear that (iv') $\nRightarrow$ (iv) unless $G$ is simply connected.

3. An annulus as an extremal region. Let $G=G(r, R)$ be an annulus $\{z: r<|z|<R, r<R\}$. Then, $A=\pi\left(R^{2}-r^{2}\right), P=2 \pi(R+r)$. So, to prove that $G(r, R)$ is an extremal domain for (2), we have to show that $\lambda(G)=R-r$. Let $\gamma_{1}=\{z:|z|=R\}, \gamma_{2}=\{z:|z|=r\}$. Put $\phi^{*}(\varsigma)=R r / z$. Then, $\phi^{*} \in A(G)$. For $\varsigma \in \gamma_{1}$, we have

$$
\left(\bar{\zeta}-\phi^{*}(\varsigma)\right) d \varsigma=\left(R e^{-i \theta}-r e^{-i \theta}\right) R i e^{i \theta} d \theta=i(R-r) d s .
$$


For $\varsigma \in \gamma_{2}$, taking into account that $\gamma_{2}$ is oriented clockwise, we obtain

$$
\left(\bar{\zeta}-\phi^{*}(\zeta)\right) d \zeta=\left(r e^{-i \theta}-R e^{-i \theta}\right)\left(-r i e^{i \theta} d \theta\right)=i(R-r) d s .
$$

Therefore, on the boundary of $G(r, R)$, the equality

$$
\left[\bar{\zeta}-\phi^{*}(\varsigma)\right] d \varsigma=i(R-r) d s
$$

holds. According to (ii) of Theorem 1, we conclude that $\lambda=R-r$.

Another way to verify this is to take the Laurent decomposition of an arbitrary $f \in A(G)$ as $f=f_{1}+f_{2}$, where $f_{1}$ is analytic in the interior of $\gamma_{1}$ and $f_{2}$ is analytic in the exterior of $\gamma_{2}$. Then, a direct calculation shows that

$$
\frac{1}{A} \iint_{G} f d A=\frac{1}{P} \int_{\gamma_{1} \cup \gamma_{2}} f d s=f_{1}(0) .
$$

So, it remains to refer to (iii) of Theorem 1.

The above example and very strong symmetric properties of extremal domains enforced by Theorem 1 lead to the following natural conjecture.

CONJECTURE. $\lambda(G)=2 A / P$ if and only if $G$ is a disk of radius $\lambda$ or an annulus $G(r, R)$ with $\lambda=R-r$.

If this conjecture is true, then Theorem 1 becomes an analytic characterization of disks and annuli.

Unfortunately, we have not been able to resolve this problem in full. In the next section we show that the conjecture does hold for simply connected regions.

4. A simply connected case. As usual,

$$
\Delta=\partial^{2} / \partial x^{2}+\partial^{2} / \partial y^{2}=4(\partial / \partial z)(\partial / \partial \bar{z})
$$

denotes the Laplace operator $(z=x+i y)$.

THEOREM 2. Let $G$ be simply connected. Then, properties (i), (ii), (iii), (iv), and (iv') of Theorem 1 are all equivalent to the following.

(a) There exists a continuous function $u$ in $G$ satisfying the Poisson differential equation

$$
\begin{cases}\Delta u \equiv-1 & \text { in } G, \text { with the boundary conditions } \\ u \equiv 0, & \partial u / \partial n \equiv c=\mathrm{const} \text { on } \Gamma .\end{cases}
$$

Here, $\partial u / \partial n=u_{n}$ denotes the normal derivative of $u$ on $\Gamma$.

(b) $G$ is a disk of radius $2|c|$.

REMARK. The implication (a) $\Rightarrow$ (b) has been proven by J. Serrin [17] for arbitrary domains $G \subset \mathbf{R}^{n}$ with $C^{2}$-boundaries, and, independently, by A. Kosmodem'yanskii [15] for convex plane domains with $C^{2}$-boundaries (also, see [18]).

PROOF. It suffices to show that (iv) of Theorem 1 implies (a) and (a) $\Rightarrow(b)$. Let us first show that (iv) $\Rightarrow$ (a). The following argument is due to Kosmodem'yanskii [15]. As $G$ is simply connected, (iv) holds for all functions harmonic in $G$ and continuous up to the boundary $\Gamma$. Set $v(\varsigma)=-\frac{1}{4}|\zeta|^{2}$. Then, $\Delta v=-1$. Define the function $v_{0}(\varsigma)$ as the solution of the Dirichlet problem in $G$ with the boundary data $\left.v_{0}\right|_{\Gamma} \equiv-v$. Set $u=v+v_{0}^{\prime}$. Then, $\Delta u=\Delta v=-1$ in $G$ and $u \equiv 0$ on $\Gamma$. Also, 
letting $f$ be an arbitrary smooth function on $\Gamma$, and denoting also by $f$ its harmonic extension to $G$, and using (iv) and Green's theorem, we obtain the following:

$\int_{\Gamma} u_{n} f d s=\int_{\Gamma}\left(u_{n} f-f_{n} u\right) d s=\iint_{G}(\Delta u f-\Delta f u) d A=-\iint_{G} f d A=-\frac{A}{P} \int_{\Gamma} f d s$.

Since $f$ was arbitrary, we conclude that $u_{n} \equiv-A / P=$ const on $\Gamma$. Thus, $u$ satisfies (a).

For the sake of completeness we include a proof of Serrin's result (a) $\Rightarrow$ (b). The following incredibly slick argument is due to $\mathrm{H}$. Weinberger [18]. The original deep argument of Serrin, combining the ideas of Steiner symmetrization and a sophisticated version of maximum principle is more involved (cf. $[\mathbf{1 5}, \mathbf{1 7}])$.

Thus, assume that (a) holds. Set $r=|\zeta|$. A direct calculation shows that

$$
\Delta\left(r \frac{\partial u}{\partial r}\right)=r \frac{\partial}{\partial r}(\Delta u)+2 \Delta u=-2
$$

in $G$. Therefore, by Green's theorem, recalling that $\left.u\right|_{\Gamma} \equiv 0$, we find that (12)

$$
\begin{aligned}
\iint_{G}\left[2 u-r \frac{\partial u}{\partial r}\right] d A & =\iint_{G}\left[-u \Delta\left(r \frac{\partial u}{\partial r}\right)+r \frac{\partial u}{\partial r} \Delta u\right] d A \\
& =\int_{\Gamma}\left[-u \frac{\partial}{\partial n}\left(r \frac{\partial u}{\partial r}\right)+r \frac{\partial u}{\partial r} \frac{\partial u}{\partial n}\right] d s=\int_{\Gamma} r \frac{\partial r}{\partial n}\left(\frac{\partial u}{\partial n}\right)^{2} d s \\
& =c^{2} \int_{\Gamma} r \frac{\partial r}{\partial n} d s=c^{2} \int_{\Gamma} 1 \cdot \frac{\partial}{\partial n}\left(\frac{1}{2} r^{2}\right) d s \\
& =c^{2} \iint_{G} \Delta\left(\frac{1}{2} r^{2}\right) d A=2 c^{2} A .
\end{aligned}
$$

Also, by Green's theorem

$$
\begin{aligned}
\iint_{G} r \frac{\partial u}{\partial r} d A & =\iint_{G} \operatorname{grad}\left(\frac{1}{2} r^{2}\right) \cdot \operatorname{grad} u d A \\
& =\int_{\Gamma} u \frac{\partial}{\partial n}\left(\frac{1}{2} r^{2}\right) d s-\iint_{G} u \Delta\left(\frac{1}{2} r^{2}\right) d A=-2 \iint_{G} u d A .
\end{aligned}
$$

Thus, we obtain from (12), (13)

$$
2 \iint_{G} u d A=c^{2} A
$$

On the other hand, we observe that

$$
1=(\Delta u)^{2} \leq 2\left[\left(\frac{\partial^{2} u}{\partial x^{2}}\right)^{2}+\left(\frac{\partial^{2} u}{\partial y^{2}}\right)^{2}\right] \leq 2\left[\left(\frac{\partial^{2} y}{\partial x^{2}}\right)^{2}+2\left(\frac{\partial^{2} u}{\partial x \partial y}\right)^{2}+\left(\frac{\partial^{2} u}{\partial y^{2}}\right)^{2}\right]
$$

Hence,

$$
\Delta\left(|\operatorname{grad} u|^{2}+u\right)=2\left[\left(\frac{\partial^{2} u}{\partial x^{2}}\right)^{2}+2\left(\frac{\partial^{2} u}{\partial x \partial y}\right)^{2}+\left(\frac{\partial^{2} u}{\partial y^{2}}\right)^{2}\right]-1 \geq 0
$$


So, $|\operatorname{grad} u|^{2}+u$ is subharmonic in $G$. Also, $|\operatorname{grad} u|^{2}+u=c^{2}$ on $\Gamma$. Therefore, according to the maximum principle, either

$$
\begin{array}{ll}
|\operatorname{grad} u|^{2}+u<c^{2} & \text { in } G, \text { or } \\
|\operatorname{grad} u|^{2}+u \equiv c^{2} & \text { in } G .
\end{array}
$$

Assuming (17) and integrating both sides of (17) over $G$, we observe from Green's theorem $(u \equiv 0$ on $\Gamma$ !) that

$$
\iint_{G}\left(|\operatorname{grad} u|^{2}+u\right) d A=-\iint_{G} u \Delta u d A+\iint_{G} u d A=2 \iint_{G} u d A<c^{2} A .
$$

But this contradicts (14) and, therefore, $|\operatorname{grad} u|^{2}+u \equiv c^{2}$ in $G$. Whence equality must hold in (16) and (15). As $\Delta u=-1$, we obtain that

$$
\frac{\partial^{2} u}{\partial x^{2}} \equiv \frac{\partial^{2} u}{\partial y^{2}} \equiv-\frac{1}{2} \quad \text { and } \quad \frac{\partial^{2} u}{\partial x \partial y} \equiv 0
$$

in $G$. Hence, up to a linear transformation, $u=-r^{2} / 4+$ const: As $\left.u\right|_{\Gamma} \equiv 0$, we conclude that $G$ is a disk.

REMARK. Condition (a) has a very natural physical interpretation. Consider a viscous incompressible fluid moving in parallel straight lines through a pipe of given cross-sectional form $G$. If we fix a $z$-axis directed along the streamlines, then the flow velocity $u$ as a function of $x, y$ alone satisfies the Poisson equation $\Delta u=-A$ in $G$, where $A$ is a constant depending on the viscosity, the density of the fluid, and on the rate of change of pressure per unit length along the pipe. The adherence condition on $G$ is $u \equiv 0$. Finally, the tangential stress per unit area on the pipe is given by $\mu(\partial u / \partial n)$, where $\mu$ is the viscosity. Thus, (a) states that the tangential stress on the pipe wall is the same at all points of the wall if and only if it has a circular cross-section (cf. [17]; also see [17] for some further discussion).

5. Concluding remarks. (I) Dr. B. Gustafsson has pointed out to the author that for simply connected domains the implication (ii) of Theorem $1 \Rightarrow$ (b) can be obtained by means of a more elementary argument. He has kindly allowed us to sketch his argument here. Differentiating (ii) with respect to a parameter $s$ we obtain

$$
\frac{d \bar{\zeta}}{d s}-i \lambda \frac{d^{2} \bar{\zeta}}{d s^{2}}=\left(\phi^{*}\right)^{\prime} \frac{d \zeta}{d s}
$$

Or, dividing both sides by $(d \bar{\zeta} / d s)$, we find

$$
1-\lambda\left(\overline{\frac{d^{2} \zeta / d s^{2}}{i d \zeta / d s}}\right)=\left(\phi^{*}\right)^{\prime}\left(\frac{d \zeta}{d s}\right)^{2} .
$$

But vectors $d^{2} \varsigma / d s^{2}$ and $d \varsigma / d s$ are orthogonal. Hence, from (18) we conclude that $\left(\phi^{*}\right)^{\prime}(d \varsigma / d s)^{2}$ is real-valued on $\Gamma$. Since $\Delta_{\Gamma} \arg (d \varsigma / d s)^{2}=4 \pi$ and $\Delta_{\Gamma} \arg \left(\phi^{*}\right)^{\prime} \geq 0$, this leads to a contradiction unless $\left(\phi^{*}\right)^{\prime} \equiv 0$, i.e. $\phi^{*} \equiv$ const. Thus, $G$ is a disk.

(II) If $G$ is multiply connected and satisfies one of the conditions (i)-(iv) of Theorem 1 and, in addition to that, $\Gamma$ contains a circular arc, then $G$ is either a disk or an annulus. 
In reality, let $\gamma \subset \Gamma$ be a circular arc of radius $R$ centered at the origin. From (ii) of Theorem 1 it follows that

$$
\phi^{*}(\zeta)=\frac{R^{2}}{\zeta}-\frac{\lambda R}{\zeta}=\frac{c}{\zeta}, \quad \text { where } c=R^{2}-\lambda R,
$$

everywhere on $\gamma$. By the uniqueness theorem $\left(\phi^{*}\right.$ is analytic!), $\phi^{*}=c / \varsigma$ in $G$. If $c=0$, then $\phi^{*} \equiv 0$ and $|\zeta| \equiv$ const on $\Gamma$. So, $G$ is a disk of radius $R$ centered at the origin.

If $c \neq 0$, from (ii) and (19) we obtain that

$$
i \lambda \varsigma \frac{d \bar{\zeta}}{d s}=|\varsigma|^{2}-c
$$

everywhere on $\Gamma$. Hence, $\operatorname{Re}\{\zeta(d \bar{\zeta} / d s)\} \equiv 0$ on $\Gamma$. But $\left.\left.d\right|_{\zeta}\right|^{2} / d s=2 \operatorname{Re}(\zeta d \bar{\zeta} / d s)$. Therefore, $|\zeta|$ locally is a constant on $\Gamma$. So, $G$ is an annulus.

This fact has also been observed by $\mathrm{B}$. Gustafsson, but his argument is quite different from the one given above. Also, a weaker version of this statement, namely that if $G$ is a circular domain which satisfies Theorem 1 , then $G$ is a disc or an annulus, has been pointed out by the referee.

(III) Let $\bar{\zeta}=S(\varsigma)$ be the Schwarz function of the boundary $\Gamma$ of $G$. So, $S(\varsigma)$ is analytic in a tubular neighborhood of $\Gamma$ and equality $\bar{\zeta}=S(\zeta)$ holds on $\Gamma$. As $d \bar{\zeta}=S^{\prime}(\varsigma) d \zeta$ on $\Gamma$ and $|d \zeta / d s| \equiv 1$,

$$
S^{\prime}(\zeta)=(d \bar{\zeta} / d s)^{2} \text {. }
$$

(For more detail on the Schwarz function we refer the reader to [4]. Also, see [1, 16].) So equation (ii) of Theorem 1 can be rewritten in the following form:

$$
S(\varsigma)-i \lambda\left(S^{\prime}(\varsigma)\right)^{1 / 2}=\phi^{*}(\varsigma), \quad \varsigma \in \Gamma \text {. }
$$

(In view of (20), there exists a single-valued branch of $\left(S^{\prime}\right)^{1 / 2}$ near $\Gamma$.) As $S, \phi^{*}$ are analytic across $\Gamma,(21)$ holds in a tubular neighborhood of $\Gamma$. Differentiating (21) and introducing a new variable $u=\left(S^{\prime}(\varsigma)\right)^{1 / 2}$, we obtain the Riccati equation

$$
u^{2}-i \lambda u^{\prime}=f, \quad \text { where } f=\left(\phi^{*}\right)^{\prime} .
$$

A standard substitution (cf. [8, Chapter XII]) $u=\left\{(-\lambda i) v^{\prime} / v\right\}$ transforms (22) into the homogeneous linear equation of the second order,

$$
v^{\prime \prime}+\frac{f}{\lambda^{2}} v=0
$$

As follows from the standard theory of ordinary differential equations in the complex domain (see [8, Chapter XII]), solutions of (23) are locally analytic in G. Hence, $u$ and $S$ are locally meromorphic in $G$. Moreover, a simple analysis of (21) and (22) shows that $u$ and $S$ can have only simple poles and all the residues of $S(z)$ are equal to $\lambda^{2}$. It seems promising to try to prove our conjecture by combining those ideas with the geometric meaning of Schwarz functions. However, we have not been able to find such a proof. The Riccati equation (21) frequently appears in certain free boundary problems concerning possible shapes of a body being in equilibrium in a potential field (see [7]). This suggests that further investigation of our problem in terms of equation (21) is desirable. 


\section{REFERENCES}

1. D. Aharanov and H. S. Shapiro, Domains on which analytic functions satisfy quadrature identities, J. Analyse Math. 30 (1976), 39-73.

2. H. Alexander, Projections of polynomial hulls, J. Funct. Anal. 3 (1973), 13-19.

3. - On the area of the spectrum of an element of a uniform algebra, Complex Approximation (B. Aupetit, ed.), Birkhäuser, Basel, 1980, pp. 3-12.

4. P. J. Davis, The Schwarz function and its applications, Carus Math. Monographs, vol. 17, (1974).

5. P. Duren, Theory of $H^{p}$-spaces, Academic Press, New York and London, 1970.

6. T. Gamelin and D. Khavinson, The isoperimetric inequality and rational approximation (in preparation).

7. P. Garabedian, On the shape of electrified droplets, Comm. Pure Appl. Math. 28 (1965), 31-34.

8. E. L. Ince, Ordinary differential equations, Longmans, Green and Co., London, New York and Toronto, 1927.

9. D. Khavinson, Annihilating measures of the algebra $R(X)$, J. Funct. Anal. 28 (1984), 175-193.

10. _ , A note on Toeplitz operators, Geometry of Banach Spaces (N. Kalton and E. Saab, eds.), Lecture Notes in Math., vol. 934, Springer-Verlag, Berlin and New York, 1986, pp. 89-95.

11. Smirnov classes of analytic functions in multiply connected domains, Appendix to English translation of Foundations of the theory of extremal problems for bounded analytic functions and their various generalizations, by S. Ya. Khavinson, Amer. Math. Soc. Transl. 129 (1986), 57-61.

12. D. Khavinson and D. Luecking, On an extremal problem in the theory of rational approximation, J. Approx. Theory (to appear).

13. S. Ya. Khavinson, Foundations of the theory of extremal problems for bounded analytic functions and their various generalizations, Amer. Math. Soc. Transl. 129 (1986), 1-57.

14. S. Ya. Khavinson and G. C. Tumarkin, On the definition of analytic functions of class $E_{p}$ in multiply connected domains, Uspekhi Mat. Nauk 13 (1958), 201-206. (Russian)

15. A. A. Kosmodem'yanskii, A converse of the mean value theorem for harmonic functions, Russian Math. Surveys 36 (1981), no. 5, 159-160; translated from Russian.

16. M. Sakai, Quadrature domains, Lecture Notes in Math., vol. 934, Springer-Verlag, Berlin and New York, 1982.

17. J. Serrin, A symmetry problem in potential theory, Arch. Rational Mech. Anal. 43 (1971), 304-318.

18. H. Weinberger, Remark on the preceeding paper of Serrin, Arch. Rational Mech. Anal. 43 (1971), 319-320.

Department of Mathematical Sciences, University of ARKansas, FayETTEVILLE, ARKANSAS 72701 